\title{
Biotin Functionalized Self-Assembled Peptide Nanofiber as an Adjuvant for Immunomodulatory Response
}

\author{
Muhammed Burak Demircan, Sehmus Tohumeken, Nuray Gunduz, \\ Mohammad Aref Khalily, Turgay Tekinay, Mustafa O. Guler,* and Ayse B. Tekinay*
}

Biotinylated peptide amphiphile (Biotin-PA) nanofibers, are designed as a noncovalent binding location for antigens, which are adjuvants to enhance, accelerate, and prolong the immune response triggered by antigens.

Presenting antigens on synthetic Biotin-PA nanofibers generated a higher immune response than the free antigens delivered with a cytosine-phosphate-guanine oligodeoxynucleotides (CpG ODN) (TLR9 agonist) adjuvant. Antigen attached Biotin-PA nanofibers trigger splenocytes to produce high levels of cytokines (IFN- $\gamma$, IL-12, TNF- $\alpha$, and IL-6) and to exhibit a superior cross-presentation of the antigen. Both Biotin-PA nanofibers and CPG ODN induce a Th-1-biased IgG subclass response; however, delivering the antigen with Biotin-PA nanofibers induce significantly greater production of total IgG and subclasses of IgG compared to delivering the antigen with CpG ODN. Contrary to CpG ODN, Biotin-PA nanofibers also enhance antigen-specific splenocyte proliferation and increase the proportion of the antigen-specific $\mathrm{CD} 8(+) \mathrm{T}$ cells. Given their biodegradability and biocompatibility, Biotin-PA nanofibers have a significant potential in immunoengineering applications as a biomaterial for the delivery of a diverse set of antigens derived from intracellular pathogens, emerging viral diseases such as COVID-19, or cancer cells to induce humoral and cellular immune responses against the antigens.

\section{Introduction}

Vaccination requires administration of antigenic materials to boost the immune system and is still the most effective strategy against infectious and intracellular pathogenic diseases. ${ }^{[1]}$ The occurrence of new pathogens, insufficient protection by application of existing vaccines, and need for effective vaccines for cancer immunotherapy require rationally engineered vaccine systems. ${ }^{[2,3]}$ Engineering synthetic subunit vaccines comprised of one or more antigenic recombinant proteins is an emerging field. There is an urging need for developing new well-defined, targeted and safe vaccines with a low cost of production. ${ }^{[4-6]}$ Due to low immunogenicity of recombinant proteins, adjuvants are used in the vaccine formulation to direct and maintain the immune response against antigens. ${ }^{[7,8]}$ The ideal adjuvant formulation should demonstrate biocompatibility and biodegradability while activating both innate and adaptive immune systems against a specific antigen. ${ }^{[7,9]}$

One of the mechanisms of action for adjuvants is their recognition as a danger
M. B. Demircan, Prof. A. B. Tekinay

Neuroscience Graduate Program

Bilkent University

Ankara 06800, Turkey

E-mail: abt@eryigit.com.tr

Dr. S. Tohumeken, Dr. N. Gunduz, Dr. M. A. Khalily, Prof. A. B. Tekinay Institute of Materials Science and Nanotechnology

Bilkent University

Ankara 06800, Turkey

Dr. N. Gunduz

The Beatson Institute for Cancer Research

Garscube Estate

Switchback Road, Bearsden, Glasgow G61 1BD, UK

Prof. A. B. Tekinay

Eryigit Research \& Development Center

Ivedik OSB, Ankara 06100, Turkey

The ORCID identification number(s) for the author(s) of this article can be found under https://doi.org/10.1002/biot.202000100

DOI: 10.1002/biot.202000100
M. B. Demircan

Department of Internal Medicine II

Hematology and Oncology

Friedrich-Schiller-University Medical Center

Jena 07743 Germany

M. B. Demircan

Leibniz-Institute on Aging

Fritz-Lipmann-Institute

Jena 07745, Germany

Dr. S. Tohumeken

Division of Hematologic Malignancies

Johns Hopkins Sidney Kimmel Comprehensive Cancer Center

Baltimore MD, USA

Prof. T. Tekinay

Gazi University

Ankara 06100, Turkey

Prof. M. O. Guler

The Pritzker School of Molecular Engineering

The University of Chicago

Chicago IL 60637, USA

E-mail: mguler@uchicago.edu 
signal by antigen-presenting cells (APCs) by activation and maturation of these cells. ${ }^{[10,11]}$ The dendritic cells (DCs) are the most important APCs and are specialized for uptake, processing, and presentation of antigens, and activation of naive T cells. ${ }^{[12]}$ While antigen presentation via the major histocompatibility complex (MHC) class II on DCs is essential for provoking antibody production by $\mathrm{B}$ cells and Th2 immune response, ${ }^{[13]}$ an effective response against infectious diseases and cancers requires Th1 immune response and activation of CD8 $+\mathrm{T}$ through association with the MHC class I complex on DCs presenting the antigen peptides via cross-presentation. ${ }^{[5]}$ Thus, an ideal adjuvant should promote the cross-presentation of the target antigens and thereby elicit a specific CD $8+T$ cell response to overcome infectious diseases and cancers.

Engineering new materials is necessary to promote antigen delivery with a depot effect and to stimulate immature APCs to enable cross-presentation by tuning the immune system properly through vaccination..$^{[5,14-16]}$ One way to design new adjuvants is to mimic pathogen cues in a reductionist fashion to manipulate intracellular pathways in APCs to enable better control of antigen presentation by changing the resultant cytokine environment and/or directing the route of antigen delivery toward crosspresentation. $^{[17]}$

Self-assembling peptides are biomaterials that have the potential to be vaccine adjuvants due to their tailorable design, chemical composition, low immunogenicity, biodegradability, and biocompatibility. ${ }^{[7]}$ These nanostructures provide a multivalent platform through display of versatile ligands with high affinity and avidity. Self-assembling peptides have been previously reported as adjuvants. ${ }^{[18-20,21]}$ However, in addition to triggering antibody production, activation of cytotoxic CD8 + $\mathrm{T}$ cells, which are necessary for the specific elimination of cells infected by viruses, intracellular bacteria, and tumors is required. ${ }^{[22]}$

In this work, we present a potent nano-adjuvant formed by biotinylated peptide amphiphile (Biotin-PA) nanofibers, which enables the presentation of biotin groups on the periphery of the nanofibers. Through streptavidin (SA)-biotin affinity, which is one of the strongest interactions known, ${ }^{[23]}$ a variety of biotinylated functional molecules are attached to the nanofibers by using SA as a linker between the nanofiber and the biotinylated antigen. ${ }^{[24]}$ In the present system, Biotin-PA nanofibers present antigens to enable internalization of the nanofibers and the antigens by the same APC for increased antigen-specific immune response. ${ }^{[25,26]}$ In addition, the structural features of Biotin-PA nanofibers such as the rod-like morphological organization and the multivalent presentation of the biotins and the antigens on the surface resemble viruses, ${ }^{[27,28]}$ and potentially trigger a Th1 immune response. We compared Biotin-PA nanofibers to cytosine-phosphate-guanine oligodeoxynucleotides (CpG ODNs), which induce a Th1-biased immune response. CpG ODNs are widely being used as adjuvants in the literature and a hepatitis B vaccine containing CpG-ODN as an adjuvant was recently approved for application in humans. ${ }^{[29]}$ Overall, we demonstrated that Biotin-PA adjuvant system conjugated to antigens improve antigen-specific humoral and cellular responses in in vitro and in vivo models and thereby can be utilized for vaccine development and immunotherapy.

\section{Results and Discussion}

\subsection{Design, Synthesis, and Characterization of the Peptide Nanofibers}

Biotin-PA molecule was designed to self-assemble into biotin presenting nanofibers (Figure 1A). The Biotin-PA molecule contains a hydrophobic lauryl group, which enables the assembly of the hydrophobic inner core of the nanofibers and a hydrophilic peptide sequence that mediates the presentation of the biotin groups on Biotin-PA nanofiber surface (Figure 1B). Between the lauryl group and the hydrophilic amino acids, hydrophobic $\beta$ sheet forming amino acids were placed to enable the formation of the nanofibers. One lysine residue was utilized to conjugate the biotin on the side chain, while another one provided positive charge for enhancing solubility at physiological $\mathrm{pH} .^{[30]}$ The PA molecules were synthesized through solid-phase peptide synthesis, purified with high-performance liquid chromatography, and analyzed by liquid chromatography-mass spectrometry (Figure S1, Supporting Information). circular dichorism (CD) spectroscopy demonstrated the dominance of $\beta$-sheet secondary structure revealed by signals at 217 and $197 \mathrm{~nm}$ (Figure 1C). ${ }^{[31]}$ Transmission electron microscopy (TEM) results showed that the Biotin-PA molecules formed virus-like cylindrical fibers with a diameter of $15.5 \pm 7.2 \mathrm{~nm}$. (Figure 1D).

\subsection{Biotinylated ovalbumin Presentation on Biotin-PA Nanofibers}

Ovalbumin (OVA) is widely used as a model antigen to explore the potential of vaccine adjuvants. ${ }^{[7]}$ We used biotinylated OVA in order to enable the binding of the OVA antigens to BiotinPA nanofibers by using SA linker which has four binding sites for biotin. The binding capability of the antigen to Biotin-PA nanofibers was analyzed by enzyme-linked immunosorbent assay (ELISA). The results showed that biotinylated OVA could efficiently bind to Biotin-PA nanofibers through SA linkers. (Figure S2A, Supporting Information).

To understand the sustained release of the antigen at the site of injection which is an important factor for the efficiency of the adjuvants, ${ }^{[32]}$ we prepared an in vivo-like environment by using Roswell Park Memorial Institute medium containing fetal bovine serum at $37^{\circ} \mathrm{C}$. Biotin-PA-coated ELISA wells were maintained in this environment for different durations. After extensive washing to remove the unattached OVA, a standard ELISA protocol was applied to analyze the amount of the remaining biotinylated OVA as bound to Biotin-PA nanofibers. The results showed that OVA release from Biotin-PA nanofibers is gradual, and thereby showing the depot effect of the nanofibers in vitro (Figure S2B, Supporting Information).

\subsection{Biotin-PA Nanofibers Enhance Cytokine Secretion}

Isolated spleen cells were utilized to analyze the immune response against Biotin-PA nanofibers in vitro. Dose-response 
A

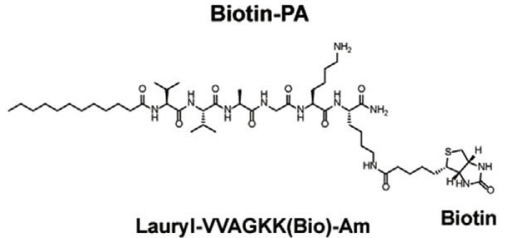

B

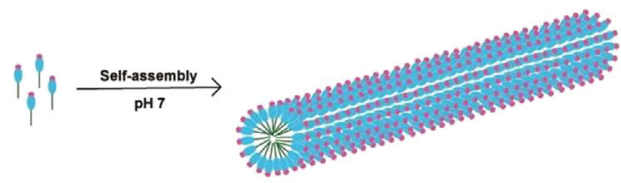

D

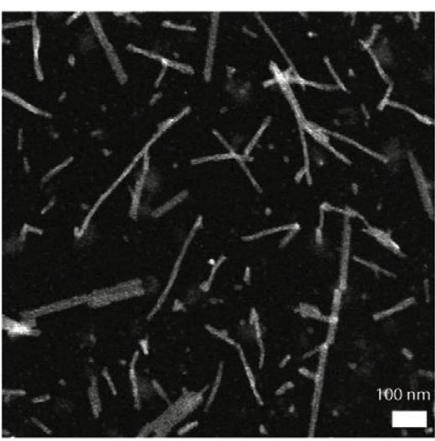

Figure 1. Characterizations of Biotin-PA nanofibers. A) The chemical structure of the Biotin-PA molecule. B) Schematic presentation of Biotin-PA nanofiber. C) CD spectra of Biotin-PA nanofibers and CPC ODN. D) STEM images of Biotin-PA nanofibers (the scale bar is $100 \mathrm{~nm}$ ).

A

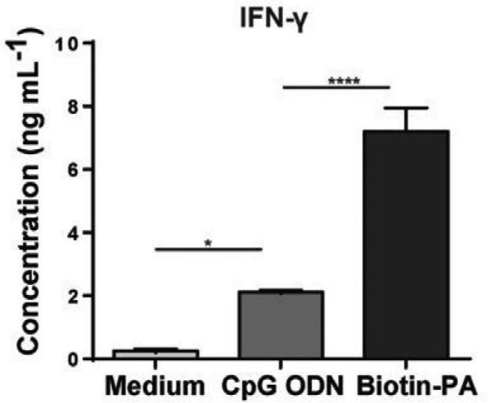

B

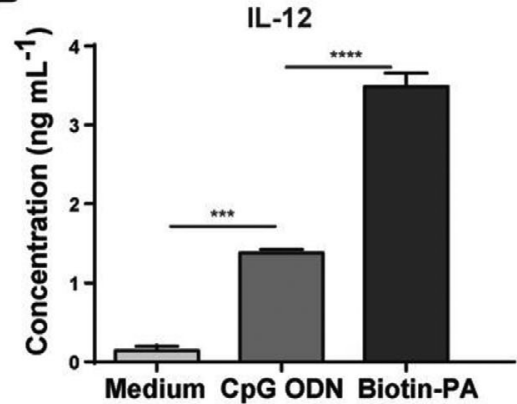

C

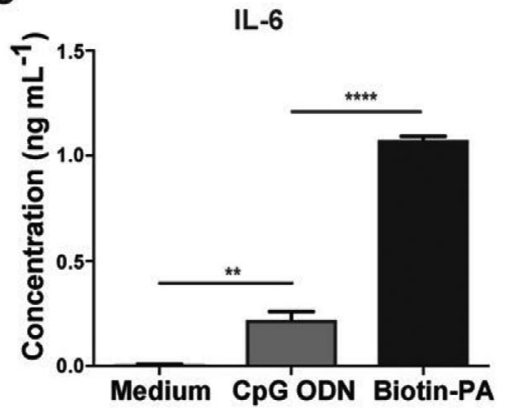

Figure 2. The effect of Biotin-PA nanofibers on cytokine production. Splenocytes were treated with CpG ODN + biotinylated OVA or Biotin-PA + SA + biotinylated OVA. A) IFN- $\gamma$, B) IL-12 and C) IL-6 concentrations in splenocyte culture supernatants were measured by ELISA. (* $* 0.05, * * p<0.01$, $* * * p<0.001$ and $* * * * p<0.0001$ according to one-way ANOVA with Tukey's Multiple Comparison post-test).

curve of the effect of Biotin-PA nanofibers on IFN- $\gamma$ production was analyzed to find an optimal dose of Biotin-PA nanofibers for the in vitro experiments (Figure S3, Supporting Information). Next, the biocompatibility of Biotin-PA nanofibers was assessed by using Alamar blue assay. CpG ODN had a proliferative effect on splenocytes, as expected, ${ }^{[27]}$ and Biotin-PA nanofibers showed good biocompatibility with splenocyte cells (Figure S4, Supporting Information). Then, the cytokine production profile of the splenocytes was analyzed in order to determine the direction of the immune response. It is known that B-class $\mathrm{CpG}$ ODN (ODN1826) triggers a Th1-biased immune response and promotes IFN- $\gamma$, IL-12, and TNF- $\alpha$ levels. ${ }^{[33]}$ Both CpG ODN and Biotin-PA nanofibers significantly promoted the levels of IFN- $\gamma$ (Figure 2A), IL-12 (Figure 2B), and TNF- $\alpha$ (Figure S5, Supporting Information); however, Biotin-PA nanofibers triggered a significantly stronger cytokine production compared to CpG ODN. In addition, Biotin-PA nanofibers, which were observed to exhibit a virus-like nanomorphology, also significantly enhanced the IL6 levels, which is known to promote the Th2 immune response and was previously shown to be a protector against viruses (Figure $2 \mathrm{C}) \cdot{ }^{\left[{ }^{[3]}\right.}$ These results suggest that Biotin-PA nanofibers can mediate a balanced Th1/Th2 phenotype, but the Th1 phenotype seems to be predominant.

\subsection{Biotin-PA Nanofibers Promote Cross-Presentation of the Antigen and Maturation of the APCs}

Cross-presentation mediates the presentation of exogenous antigens onto MHC class I molecules, which is vital for CD8 + T cell activation. ${ }^{[35]}$ Therefore, an ideal adjuvant should promote the cross-presentation of the co-delivered antigens in order to 

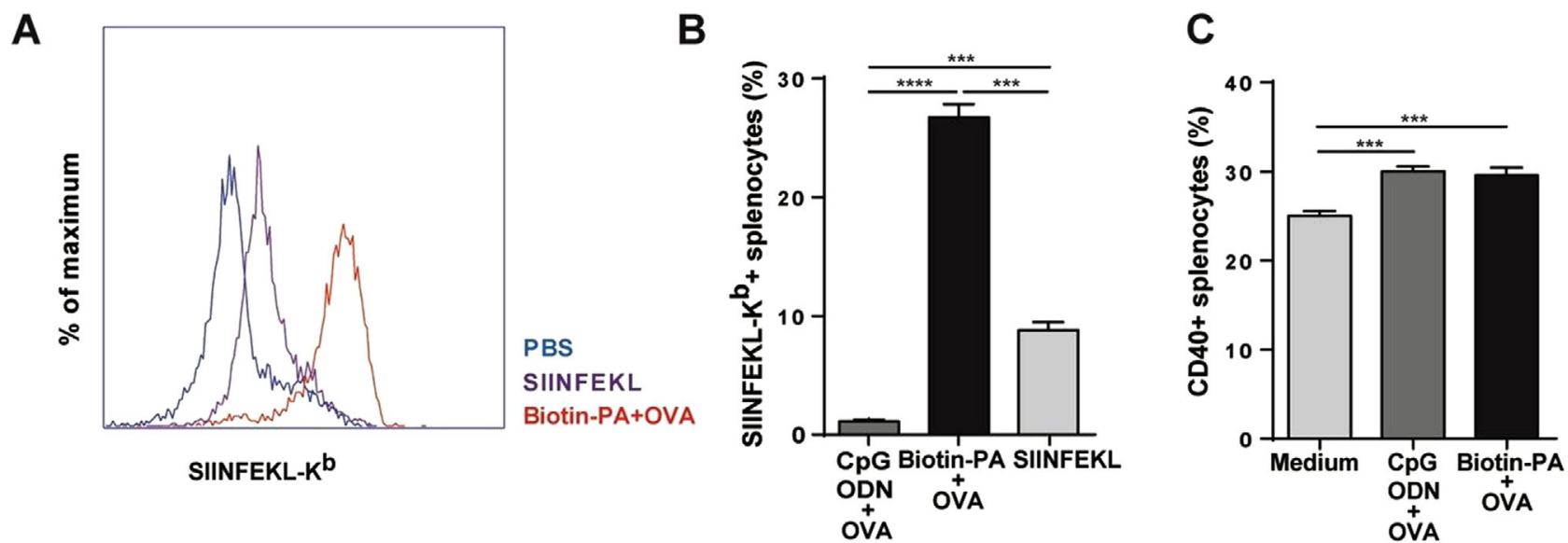

Figure 3. The effect of Biotin-PA nanofibers on antigen presentation and maturation of splenocytes. Splenocytes were treated with CpG ODN + biotinylated OVA or Biotin-PA + SA + biotinylated OVA for $24 \mathrm{~h}$. A,B) the extent of cross-presentation of OVA was measured using an antibody that recognizes SIINFEKL complexed with $\mathrm{H}-2 \mathrm{~K}^{\mathrm{b}}$, and $\mathrm{C}$ ) the percentage of cells expressing CD40 was assessed using flow cytometry. ( $* * * p<0.001$ and $* * * * p<0.0001$ according to one-way ANOVA with Tukey's multiple comparison post-test).

eradicate infected cells or cancer cells through the activation of CD8 + T cells. In order to investigate whether Biotin-PA nanofibers have the capability to promote cross-presentation, splenocytes were treated with "CpG ODN + biotinylated OVA" or "Biotin-PA nanofibers + SA + biotinylated OVA" or "SIINFEKL peptides" as a positive control. SIINFEKL is a well-characterized CD8 + T cell epitope found in OVA. For this purpose, cells were stained with a monoclonal antibody specific for SIINFEKL complexed with $\mathrm{H}-2 \mathrm{~K}^{\mathrm{b}}$, and the percentage of SIINFEKL-bound, MHC class I carrying cells was analyzed using flow cytometry. ${ }^{[36]}$ As shown in Figure 3B, Biotin-PA nanofibers promoted the presentation of the SIINFELK epitope. Cross-presentation is the only way of presenting exogenous antigens onto MHC class I. Since the level of SIINFEKL presentation is an indicator of the total OVA presentation, this result suggests that Biotin-PA nanofibers enhance antigen-specific cross-presentation.

The upregulation of the co-stimulatory molecules on the immune cells is a sign of the immune activation that leads APC maturation and APC-mediated antigen presentation. It is known that pathogenic signals such as CpG ODN enables the upregulation of co-stimulatory molecules on the immune cells. ${ }^{[27]}$ To verify whether Biotin-PA nanofibers have the capability to promote APC maturation, splenocytes were used as APCs since only APCs in the cell pool are able to do antigen presentation and have CD40 expression. Splenocytes were cultured in the presence of $\mathrm{CpG}$ ODN or Biotin-PA nanofibers. The expression levels of the cell surface marker CD40 was analyzed using flow cytometry. The results revealed that both $\mathrm{CpG}$ ODN and Biotin-PA nanofibers significantly enhanced the expression of CD40 (Figure 3C), showing that Biotin-PA nanofibers enhance co-stimulatory molecule expression and thereby induce APC maturation.

\subsection{The Effects of Biotin-PA Nanofibers on Humoral Immunity}

Biotin-PA nanofibers showed a remarkable potential for triggering an immune response in in vitro experiments; thus, next, we assessed its potential for being used as an adjuvant in vivo. BALB/c mice were subcutaneously immunized twice (day 0 and 15) with CpG ODN + biotinylated OVA or Biotin-PA nanofiber + SA + biotinylated OVA. Blood samples were collected at day 21. Since the antibody titer against the co-delivered antigen is a significant parameter for assessing the activation of humoral immune responses, OVA-specific IgG levels were analyzed from the isolated sera. As shown in Figure 4A, total IgG levels were significantly boosted by Biotin-PA nanofibers, compared to the CpG ODN adjuvant, suggesting that Biotin-PA nanofibers are superior to $\mathrm{CpG}$ ODN for triggering an antigen-specific humoral immune response.

In order to determine the influence of Biotin-PA nanofibers on IgG isotype switching, we then analyzed the levels of IgG1 and IgG2a. The results showed that the mice vaccinated with BiotinPA nanofibers had significantly higher OVA-specific IgG1 and IgG2a antibody production compared to those vaccinated with the CpG ODN group (Figure 4B,C).

When the IgG2a/IgG1 ratios were compared, both CpG ODN and Biotin-PA nanofibers had an IgG2a/IgG1 ratio greater than 1 , which indicates that the Th1 phenotype is predominant for both of them (Figure 4D). Overall, Biotin-PA nanofibers mediated a balanced Th1/Th2 phenotype, even though the Th1 phenotype was predominant, ${ }^{[37]}$ and these results are consistent with prior results obtained from the cytokine release profile analysis of splenocytes (Figure 2A-C).

\subsection{The Effects of Biotin-PA Nanofibers on Cell-Mediated Immune Response}

The cellular immunity is the most important line of an effective immune response against infected cells or tumor cells, and the functional role of a vaccine is enabling to trigger a strong and rapid cellular immunity against the same pathogen after the second exposure. ${ }^{[38]}$ To analyze whether Biotin-PA nanofibers affect cellular immune response, single-cell suspensions of 
A

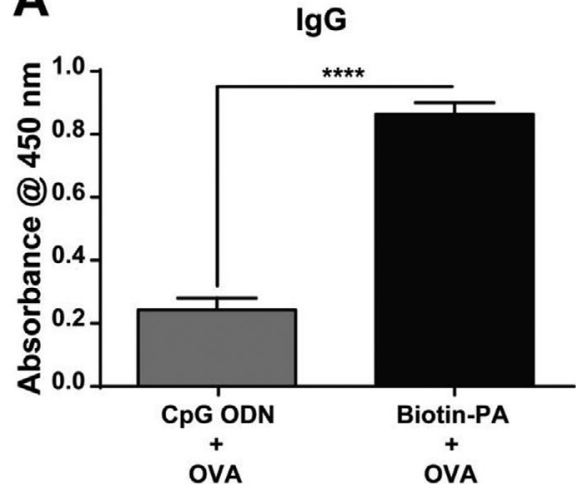

C

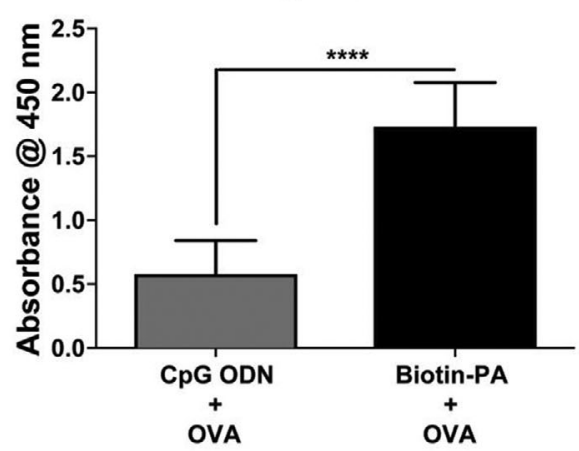

B

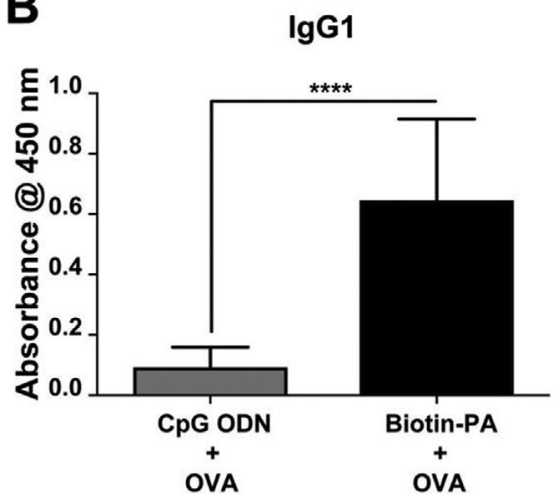

D

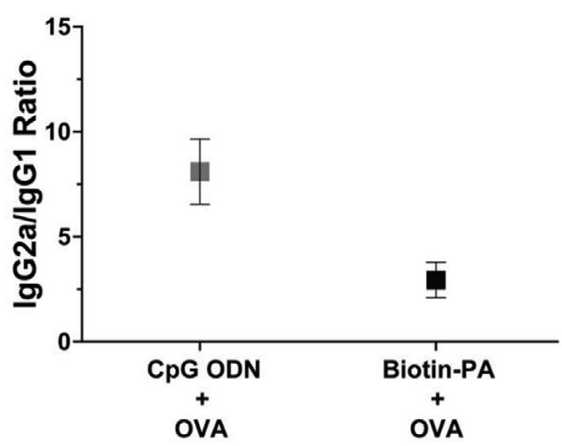

Figure 4. The effect of Biotin-PA nanofibers on OVA-specific antibody production and antibody isotype switching. A) 1 week after the second injection, IgG levels ( $1 / 10$ diluted sera) were measured with ELISA. B) The IgG 1 and C) IgG2a levels (1/100 diluted sera) were measured with ELISA. D) The ratio of $\operatorname{lgG} 2 \mathrm{a} / \lg \mathrm{l} 1$ is shown. (***** $p<0.0001$ according to Student's $t$-test).

lymphocytes were isolated from the spleens of immunized mice (1 week after the second injection with CpG ODN + biotinylated OVA or Biotin-PA nanofibers + SA + biotinylated OVA). These splenocytes were re-stimulated with OVA, and the proliferation of re-stimulated cells was analyzed with BrdU assay and fluorescence activated cell sorting (FACS) analysis. BrdU assay results showed that Biotin-PA nanofibers promoted splenocyte proliferation by twofold if the splenocytes were re-stimulated with OVA. However, there was no proliferation in CpG ODN groups after re-stimulation with OVA (Figure 5A). Therefore, Biotin-PA nanofibers were found to be superior to the CpG ODN in promoting OVA-specific splenocyte proliferation.

CD8 + T cells are critically important in order to recognize and specifically destroy non-normal cells such as infected cells or tumor cells. In order to investigate the effect of BiotinPA nanofibers on antigen specific CD8 + T cells formation, splenocytes that were isolated from immunized mice were re-stimulated with OVA. These cells were then stained with anti-CD8a-FITC antibodies. As shown in Figure 5C, Biotin-PA nanofibers enhanced CD8 $+\mathrm{T}$ cell proliferation significantly more than CpG ODN group. This result is consistent with the results of the cytokine release (Figure 2A-C) and SIINFEKL presentation (Figure 3B) analyses since these are required for CD8 $+\mathrm{T}$ cell activation. Overall, these results suggest that BiotinPA nanofibers elicit higher frequencies of OVA-specific CD8
$+\mathrm{T}$ cells by directing the immune response toward Th1, and thereby could be used to regulate the immune responses in order to eliminate infected cells or tumor cells.

\section{Conclusion}

Over the past decade, subunit vaccines have become the most popular formulation for modern vaccines because of their advantages in terms of safety, ease of manufacture, and precise immune targeting. ${ }^{[39]}$ Immense potential for the rapid development and production of an effective vaccine against emerging diseases is making them more attractive strategy for vaccine design. Peptide-based self-assembled nanostructures are one of the major class among the emerging subunit vaccines in recent years and have been shown as capable to elicit long-lasting antibody titer, ${ }^{[40,41]}$ induce $\mathrm{CD}^{+} \mathrm{T}$ cell responses, ${ }^{[42,43]}$ enhance the antitumor immune response, ${ }^{[44,45]}$ provide protection against bacterial or viral infections. ${ }^{[43,46,47]}$ Interestingly, a biotin-SA interaction based, pathogen-specific, self-assembled subunit vaccine system has been generated and pre-clinically tested in less than 120 days, ${ }^{[48]}$ and is currently being used as a differentiated, rapid approach to finding a COVID-19 vaccine. Thus, in newly emerging pandemic, for rapid production of an effective vaccine, subunit vaccines and peptide-based self-assembled vaccine systems are promising candidates. ${ }^{[49]}$ We reported use of 


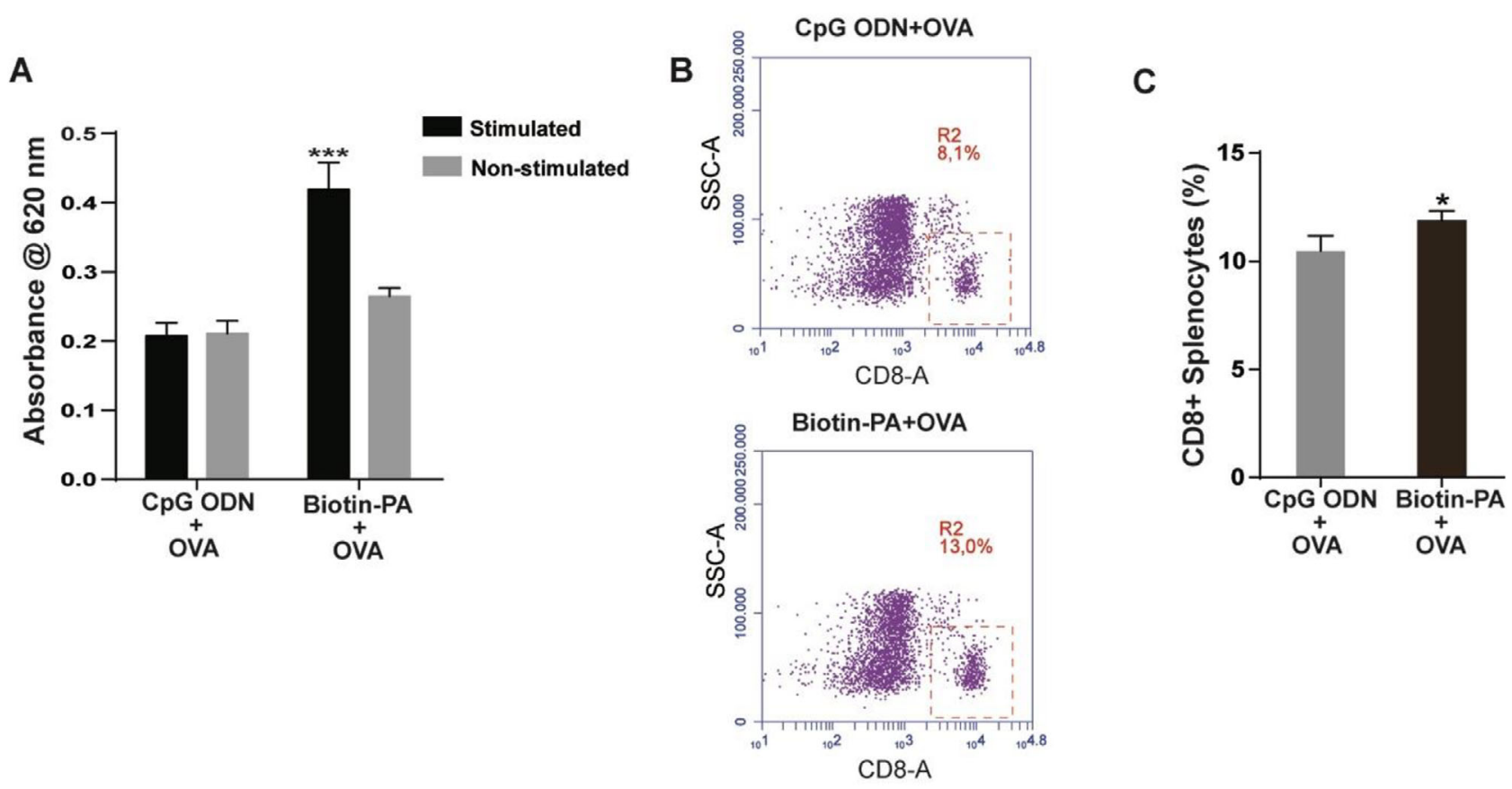

Figure 5. The effect of Biotin-PA nanofibers on OVA-specific splenocyte proliferation and CD8 + T cell proliferation. A) After ex vivo OVA re-stimulation, splenocyte proliferation was assessed using BrdU assays. The results demonstrated the level of proliferated splenocytes. B) FACS analysis of CD $8+T$ cell populations. After ex vivo OVA re-stimulation, CD8 + T cell proliferation was assessed using FACS analysis. C) The cumulative results demonstrated the percentages of CD8 + T cells after ex vivo OVA re-stimulation. (* $p<0.05$, $* * * * p<0.001$ according to Student's $t$-test)

self-assembled Biotin-PA nanofibers as a potent adjuvant that presents antigens on the surface for triggering humoral and cellular immune responses. We observed that Biotin-PA molecules formed nanofibers, and biotinylated OVA antigens were attached on these nanofibers through noncovalent, biotin-SA-biotin interactions, which enabled immobilization of the antigens on the surface of the nanofibers similar to the natural pattern of rodshaped viruses. Biotin-PA nanofibers strongly enhanced the secretion of some important cytokines such as IFN- $\gamma$, IL-12, and IL-6, the maturation of APCs, and cross-presentation of OVA. We observed that Biotin-PA nanofibers strongly induced antigenspecific antibody production, and proliferation of CD8 + T cells by directing the route of immunity toward Th1 phenotype in vivo. Interestingly, Biotin-PA nanofibers were more effective than $\mathrm{CpG}$ ODN adjuvants in triggering both humoral and cellular immune response, which shows that these nanofibers provide a promising immunotherapeutic agent for inducing antigen-specific immune response. Overall, these results show the significant potential of Biotin-PA nanofibers as vaccine adjuvants. Various biotinylated or SA-conjugated antigens can be easily attached to Biotin-PA nanofibers, enabling this synthetic self-assembled peptide nanofiber to be used as an antigen delivery vehicle in vaccines to generate a strong adjuvant effect and to elicit a Th1based immune response specific for the target antigens. Furthermore, using Biotin-PA nanofibers in vaccines enables synthetic production of vaccines, which should increase safety and reduce the need for cold chains for carriage and storage of the vaccines. However, future investigations are necessary to understand the molecular mechanism of action for Biotin-PA nanofibers as a potent adjuvant.

\section{Experimental Section}

Materials: Rink amide MBHA resin, 9-fluorenylmethoxycarbonyl (Fmoc), tert-butoxycarbonyl (Boc), and 2-(1H-benzotriazol-1-yl)-1,1,3,3tetramethyluronium hexafluorophosphate (HBTU) were obtained from $A B C R$ and NovaBiochem. All other chemicals for peptide synthesis were purchased from Merck or VWR. The cell culture reagents were purchased from Life Technologies, while the non-essential amino acid solution was from Sigma Aldrich. ODN 1826 (a class B CpG ODN) was purchased from Invivogen. The Alamar Blue cell viability reagent was purchased from Invitrogen. CD40 and OVA257-264 (SIINFEKL) peptide bound to $\mathrm{H}$ $2 \mathrm{~Kb}$ APC antibodies for flow cytometry were purchased from eBioscience. The ELISA reagents were from Life Technologies. IFN- $\gamma$ antibodies, IL12 antibodies, and IFN- $\gamma$ protein were purchased from R\&D systems. IL- 6 antibody and protein were obtained from eBioscience. The OVAspecific antibodies IgG2a (Abcam), IgG 1, IgG2b (eBioscience), and antimouse IgG-Peroxidase antibody (Sigma Aldrich) were provided by Southern Biotechnologies (USA). BrdU (5-bromo-2-deoxyuridine) was obtained from Thermo Scientific.

Peptide Synthesis: The synthesis of lauryl-VVAGKK-Biotin-Am (BiotinPA) was performed on Rink Amide MBHA resin. Three equivalents of N,Ndiisopropylethylamine, 1.95 equivalents of HBTU, and 2 equivalents of Fmoc-protected amino acid were used for amino acid couplings for $3 \mathrm{~h}$. A $5 \%(\mathrm{v} / \mathrm{v})$ solution of trifluoroacetic acid (TFA) in dichloromethane (DCM) was used to remove the Mtt group of Fmoc-Lys (Mtt)-OH, and then biotin conjugation was carried out using the same protocol as for the amino acid couplings. A solution of $20 \%(\mathrm{v} / \mathrm{v}$ ) piperidine in dimethylformamide (DMF) was used to remove the Fmoc group for $20 \mathrm{~min}$. Then, a 10\% (v/v) acetic anhydride solution in DMF was used to block the remaining free amine groups for $30 \mathrm{~min}$. The resin was washed with a mixture of DMF, $\mathrm{DCM}$, and DMF after each step. Removal of the peptide from the resins was accomplished by using a TFA/triisopropyl silane/ $\mathrm{H}_{2} \mathrm{O} / D C M$ mixture (with a ratio of 5:2.5:2.5:90). The peptide solution was concentrated by a rotary evaporator followed by addition of cold ether to obtain a white precipitate. The peptide was acquired after freeze-drying. 
Preparation of the Antigen-Coupled Biotin-PA Nanofibers: Biotin-PA nanofibers were prepared through the spontaneous self-assembly of $15 \mu \mathrm{L}$ of $0.023 \%(w / v)$ Biotin-PA molecules in an aqueous environment. The self-assembled nanofibers were mixed with SA (Sigma-Aldrich), and after $40 \mathrm{~min}$, biotinylated OVA (Galab) was added to form the nanostructure (the molar ratio for Biotin-PA nanofiber, SA, and OVA was 1:1:1).

TEM Imaging: Biotin-PA nanofibers were imaged by TEM. $50 \mu \mathrm{L}$ of the solution was prepared on a parafilm. The TEM grids were exposed to the solutions and then withdrawn. $2 \%(\mathrm{w} / \mathrm{v})$ uranyl acetate was used to stain Biotin-PA nanofibers. After washing with $\mathrm{dd}_{2} \mathrm{O}$ twice, the grids were dried for $1 \mathrm{~h}$ at room temperature. Images were taken with a FEI Tecnai G2F30 instrument in STEM mode.

Circular Dichroism Spectroscopy: $0.2 \mathrm{~mm}$ solution of Biotin-PA nanofibers were measured from 300 to $190 \mathrm{~nm}$. (Scanning speed $=100 \mathrm{~nm}$ $\min ^{-1}$, DIT $=4 \mathrm{~s}$, bandwidth $=1 \mathrm{~nm}$ and the data pitch $=1 \mathrm{~nm}$ ). All measurements were performed with three data accumulations, and sensitivity was chosen as the standard. A JASCO J815 CD spectrometer was used for the data accumulations.

Cytokine Analysis through ELISA: Splenocytes were cultured in 96-well culture plates $\left(5 \times 10^{5}\right.$ cells per well). $15 \mu \mathrm{L}$ of $0.023 \%(\mathrm{w} / \mathrm{v})$ Biotin-PA were mixed with SA ( $181 \mathrm{ng} \mathrm{mL}^{-1}$ ) and incubated for $30 \mathrm{~min}$ at $\mathrm{RT}$, and followed with incubation of biotinylated OVA (137 $\left.\mathrm{ng} \mathrm{mL}^{-1}\right)$ for 30 min at RT. Formed Biotin-PA nanofibers and CPC ODN $\left(1 \mu \mathrm{g} \mathrm{mL}^{-1}\right)+$ biotinylated OVA (137 $\mathrm{ng} \mathrm{mL}^{-1}$ ) were used to induce murine splenocyte cytokine secretion as shown in a previous study. ${ }^{[29]} \mathrm{After} 48 \mathrm{~h}$, the supernatants were collected and transferred to MaxiSorp plates (Thermo Scientific, NUNC), which were coated with IL-6, IL-12 or IFN- $\gamma$ capture antibodies and blocked with $1 \times$ assay buffer and stored at $4{ }^{\circ} \mathrm{C}$ overnight. After five washes with washing buffer and drying, biotin-labeled detection antibodies were added to the wells and incubated for $2 \mathrm{~h}$ at room temperature. After another five washes with washing buffer and drying, HRP (horseradish peroxidase)conjugated SA was added to the wells and incubated for $\mathrm{th}$ at room temperature. The samples were washed again five times with washing buffer and dried, after which 3,3',5,5'-Tetramethylbenzidine substrate was added to the wells and incubated for $20 \mathrm{~min}$. Then, stop solution $\left(1.8 \mathrm{~N} \mathrm{H}_{2} \mathrm{SO}_{4}\right)$ was added, and absorbance was measured with a microplate reader (Spectramax M5, Molecular Devices) at $450 \mathrm{~nm}$ (with a subtracted reference value of $650 \mathrm{~nm}$ ). All treatments were performed for at least three replicates and are shown as means $+/-$ standard deviation.

Analyses of Surface Co-stimulatory Markers and SIINFEKL H-2Kb Complexes: For analyses of the expression levels of CD40 co-stimulatory molecules and the levels of the SIINFEKL presentation, specific mAbs were used to stain CD40 and SIINFEKL complexed with $\mathrm{H}-2 \mathrm{~K}^{\mathrm{b}}$. Splenocytes were seeded into 96 -well culture plates $\left(5 \times 10^{5}\right.$ cells per well), and then CpG ODN $\left(1 \mu \mathrm{g} \mathrm{mL}^{-1}\right)+$ OVA (137 $\left.\mathrm{ng} \mathrm{mL}^{-1}\right)$ and Biotin-PA nanofibers $\left(15 \mu \mathrm{L}\right.$ of $0.023 \%(\mathrm{w} / \mathrm{v})$ Biotin-PA) + SA $\left(181 \mathrm{ng} \mathrm{mL}^{-1}\right)+$ biotinylated OVA (137 $\mathrm{ng} \mathrm{mL}^{-1}$ ) (prepared as described above) were added to the wells. After $24 \mathrm{~h}$, the cells were transferred to Eppendorf tubes, centrifuged, and washed with $1 \times$ phosphate buffered saline (PBS). After blocking with FACS buffer ( $3 \%$ BSA + $1 \%$ sodium azide in $1 \times$ PBS) for 10 min, specific mAbs were added to the Eppendorf tubes and incubated for $1 \mathrm{~h}$ at $4{ }^{\circ} \mathrm{C}$. After washing with $1 \times$ PBS, the cells were analyzed by flow cytometry (Accuri C6-Cytometer equipment with BD Accuri C6 software). The experiments were performed with at least three replicates and two independent experiments.

Animals: All animal experiments were approved by the Animal Ethics Committee of Gazi University Hospital (Protocol G.U.ET-15.063). Primary spleen cells were isolated from adult C57BL/6 (only used for SIINFEKL presentation analysis) and 10-14 weeks old BALB/C mice, which were housed under controlled conditions with free access to food and water.

Immunization and Determination of Antibody Response: Male BALB/C mice (10-13 weeks old) were immunized subcutaneously with $500 \mu \mathrm{L}$ vaccine formulations (in isotonic sucrose solution). Since it was well established that OVA was only weakly immunogenic and thereby do not elicit a strong immune response by itself, $[5,27]$ only OVA group was excluded and the mice were divided into two groups $(n=5)$ and immunized with CpG ODN $(10 \mu \mathrm{g})+$ biotinylated OVA $(6.8 \mu \mathrm{g})$, as a positive control, and formed Biotin-PA nanofibers (prepared as described above by using Biotin-
PA $(150 \mu \mathrm{g})+\mathrm{SA}(9.2 \mu \mathrm{g})+$ biotinylated OVA $(6.8 \mu \mathrm{g})$. The mice were immunized two times, at day 0 and 15 , and sacrificed on day 21 . On day 21 , the animals were bled, and sera were obtained. The amount of IgGs in the sera was assessed with ELISA. OVA antigen was coated onto MaxiSorp plates and incubated at $4{ }^{\circ} \mathrm{C}$ overnight. The next day, the wells were blocked with $1 \times$ assay buffer, and the diluted sera were added to the wells and stored at $4{ }^{\circ} \mathrm{C}$ overnight. After five washes with washing buffer and drying, the IgGs were detected with HRP-conjugated anti-lgG, anti-lgG 1 and antiIgG2a. After the addition of TBM substrate, absorbance was measured with a microplate reader (SpectramaxM5) at $450 \mathrm{~nm}$ (with a subtracted reference value of $650 \mathrm{~nm}$ ).

Splenocyte Proliferation Assay: Splenocytes of the immunized mice were stimulated by OVA, and splenocyte proliferation was analyzed using BrdU assay (Roche). The cells were seeded into 96-well culture plates $\left(5 \times 10^{5}\right.$ cells per well). Then, $10 \mu \mathrm{M}$ BrdU labeling solution was added to the wells and incubated for $24 \mathrm{~h}$. The next day, the splenocytes were fixed with FixDenat for 30 min. Anti BrdU-POD solution was added, and the mixture was incubated for $90 \mathrm{~min}$. After the substrate solution was added, absorbance was measured by a microplate reader (SpectramaxM5) at $370 \mathrm{~nm}$ (with a subtracted reference value of $492 \mathrm{~nm}$ ). Absorbance value indicated the proliferation rates of the cells relative to those of untreated cells.

CD8 + T Cell Proliferation Assay: The splenocytes of the immunized mice were seeded into 96 -well culture plates $\left(5 \times 10^{5}\right.$ cells per well), and stimulated by OVA for $48 \mathrm{~h}$. After the simulation, the cells were stained with antibodies for surface markers (anti-CD8a-FITC antibodies from eBioscience). The stained cells (CD8 $+T$ cells) were analyzed using flow cytometry (Accuri C6-Cytometer equipment with BD Accuri C6 software)

Statistical Analysis: Statistical analysis was performed using Graphpad Prism 7. One-way ANOVA, and Student's t-test were used to compare the groups. The error bars show the SEM (standard error of mean), and a $p$ value of less than 0.05 was accepted as statistically significant.

\section{Supporting Information}

Supporting Information is available from the Wiley Online Library or from the author.

\section{Acknowledgements}

M.B.D. and S.T. contributed equally to this work. The authors would like to thank TEM specialist Mr. M. Guler for technical help in TEM imaging. S.T. was supported by TUBITAK BIDEB-2210 M.Sc. fellowship. N.G. and A.K. were supported by TUBITAK BIDEB-2211 PhD fellowship. This work was partially supported by the Scientific and Technological Research Council of Turkey (TUBITAK 114Z562). A.B.T. acknowledges support from the Science Academy Outstanding Young Scientist Award (BAGEP).

\section{Conflict of Interest}

The authors declare no conflict of interest.

\section{Keywords}

adjuvant, peptide nanofiber, self-assembly, vaccine, virus-like particles

Received: March 23, 2020 Revised: June 15, 2020 Published online: July 26, 2020 
[1] S. Lee, M. T. Nguyen, Immune Network 2015, 15, 51.

[2] F. Mosca, E. Tritto, A. Muzzi, E. Monaci, F. Bagnoli, C. lavarone, D. O'Hagan, R. Rappuoli, E. De Gregorio, Proc. Natl. Acad. Sci. USA 2008, 105, 10501.

[3] K. Palucka, J. Banchereau, Interaction of Immune and Cancer Cells, Springer, Heidelberg, 2014, pp. 75.

[4] Z. Liu, H. Zhou, W. Wang, W. Tan, Y. X. Fu, M. Zhu, Sci. Rep. 2014, 4, 7266.

[5] J. J. Moon, H. Suh, A. Bershteyn, M. T. Stephan, H. Liu, B. Huang, M. Sohail, S. Luo, S. H. Um, H. Khant, J. T. Goodwin, J. Ramos, W. Chiu, D. J. Irvine, Nat. Mater. 2011, 10, 243.

[6] S. G. Reed, S. Bertholet, R. N. Coler, M. Friede, Trends Immunol. 2009, 30, 23.

[7] H. Wang, Z. Luo, Y. Wang, T. He, C. Yang, C. Ren, L. Ma, C. Gong, X Li, Z. Yang, Adv. Funct. Mater. 2016, 26, 1822.

[8] C. Foged, Ther. Delivery 2011, 2, 1057.

[9] G. Verdeil, K. Marquardt, C. D. Surh, L. A. Sherman, Proc. Natl. Acad. Sci. USA 2008, 105, 16683.

[10] S. Awate, L. A. Babiuk, G. Mutwiri, Front. Immunol. 2013, 4, 114.

[11] Y. Dang, W. M. Wagner, E. Gad, L. Rastetter, C. M. Berger, G. E. Holt, M. L. Disis, Clin. Cancer Res. 2012, 18, 3122.

[12] F. A. Sharp, D. Ruane, B. Claass, E. Creagh, J. Harris, P. Malyala, M. Singh, D. T. O'Hagan, V. Pétrilli, J. Tschopp, L. A. J. O'Neill, E. C. Lavelle, Proc. Natl. Acad. Sci. USA 2009, 106, 870.

[13] K. Inaba, R. M. Steinman, J. Exp. Med. 1984, 160, 1717.

[14] A. K. Salem, Methods Mol. Biol. https://doi.org/10.1007/978- 14939-6445-1_14. Springer, Heidelberg, 2017, 1494, 201. https://link. springer.com/protocol/10.1007\%2F978-1-4939-6445-1_14

[15] M. Dierendonck, K. Fierens, R. De Rycke, L. Lybaert, S. Maji, Z. Zhang, Q. Zhang, R. Hoogenboom, B. N. Lambrecht, J. P. J. Grooten Remon, S. De Koker, B. G. De Geest, Adv. Funct. Mater. 2014, 24, 4634.

[16] D. Li, Kordalivand, N., Fransen, M. F., Ossendorp, K. Raemdonck, T. Vermonden, W. E. Hennink, C. F. van Nostrum, Adv. Funct. Mater. 2015, 25, 2993.

[17] J. A. Hubbell, S. N. Thomas, M. A. Swartz, Nature 2009, 462, 449.

[18] J. S. Rudra, Y. F. Tian, J. P. Jung, J. H. Collier, Proc. Natl. Acad. Sci. USA 2010, 107, 622.

[19] A. Trent, B. D. Ulery, M. J. Black, J. C. Barrett, S. Liang, Y. Kostenko, N. A. David, M. V. Tirrell, AAPS J. 2015, 17, 380.

[20] J. S. Rudra, T. Sun, K. C. Bird, M. D. Daniels, J. Z. Gasiorowski, A. S. Chong, J. H. Collier, ACS Nano 2012, 6, 1557.

[21] G. A. Hudalla, J. A. Modica, Y. F. Tian, J. S. Rudra, A. S. Chong, T. Sun, M. Mrksich, J. H. Collier, Adv. Healthcare Mater. 2013, 2, 1114.

[22] D. C. Tscharke, N. P. Croft, P. C. Doherty, N. L. La Gruta, Nat. Rev. Immunol. 2015, 15, 705.

[23] C. E. Chivers, A. L. Koner, E. D. Lowe, M. Howarth, Biochem. J. 2011, 435, 55.

[24] S. Chattopadhaya, L. P. Tan, S. Q. Yao, Nat. Protoc. 2006, 1, 2386.
[25] T. J. Moyer, A. C. Zmolek, D. J. Irvine, J. Clin. Invest. 2016, 126, 799.

[26] S. Tohumeken, N. Gunduz, M. B. Demircan, G. Gunay, A. E. Topal, M. A. Khalily, T. Tekinay, A. Dana, M. O. Guler, A. B. Tekinay, Adv. Biosyst. 2017, 1, 1700015.

[27] R. Mammadov, G. Cinar, N. Gunduz, M. Goktas, H. Kayhan, S. Tohumeken, A. E. Topal, I. Orujalipoor, T. Delibasi, A. Dana, S. Ide, A. B. Tekinay, M. O. Guler, Sci. Rep. 2015, 5, 16728.

[28] G. P. Pijlman, Biotechnol. J. 2015, 10, 659.

[29] L. M. Splawn, C. A. Bailey, J. P. Medina, J. C. Cho, Drug Today 2018, 54, 399.

[30] H. Cui, M. J. Webber, S. I. Stupp, Peptide Sci. 2010, 94, 1.

[31] M. O. Guler, R. C. Claussen, S. I. Stupp, J. Mater. Chem. 2005, 15, 4507.

[32] B. Pulendran, R. Ahmed, Nat. Immunol. 2011, 12, 509.

[33] J. Vollmer, R. Weeratna, P. Payette, M. Jurk, C. Schetter, M. Laucht, T. Wader, S. Tluk, M. Liu, H. L. Davis, A. M. Krieg, Eur. J. Immunol. 2004, 34, 251.

[34] O. Dienz, J. G. Rud, S. M. Eaton, P. A. Lanthier, E. Burg, A. Drew, J. Bunn, B. T. Suratt, L. Haynes, M. Rincon, Mucosal Immunol. 2012, 5, 258.

[35] O. P. Joffre, E. Segura, A. Savina, S. Amigorena, Nat. Rev. Immunol. 2012, 12, 557.

[36] P. D. Hulseberg, A. Zozulya, H. H. Chu, J. A. Triccas, Z. Fabry, M. Sandor, Immunol. Lett. 2010, 130, 36.

[37] D. Yadav, G. Khuller, Immunol. Cell Biol. 2001, 79, 207.

[38] Y. Cao, Y. Ma, M. Zhang, H. Wang, X. Tu, H. Shen, J. Dai, H. Guo, Z. Zhang, Adv. Funct. Mater. 2014, 24, 6963.

[39] A. Depelsenaire, M. Kendall, P. Young, D. Muller, Micro and Nanotechnology in Vaccine Development, Elsevier, Heidelberg, 2017, pp. 47.

[40] C. Yang, F. Shi, C. Li, Y. Wang, Z. Yang, ACS Biomater. Sci. Eng. 2017, 4, 2000.

[41] J. S. Rudra, S. Mishra, A. S. Chong, R. A. Mitchell, E. H. Nardin, V. Nussenzweig, J. H. Collier, Biomaterials 2012, 33, 6476.

[42] C. B. Chesson, E. J. Huelsmann, A. T. Lacek, F. J. Kohlhapp, M. F. Webb, A. Nabatiyan, A. Zloza, J. S. Rudra, Vaccine 2014, 32, 1174.

[43] M. A. Files, J. S. Rudra, M. Endsley, J. Immnol. 2019, 202, 139.2.

[44] Z. Wang, Y. Shang, Z. Tan, X. Li, G. Li, C. Ren, F. Wang, Z. Yang, J. Liu, Theranostics 2020, 10, 657.

[45] C. B. Chesson, M. Huante, R. J. Nusbaum, A. G. Walker, T. M. Clover, J. Chinnaswamy, J. J. Endsley, J. S. Rudra, Sci. Rep. 2018, 8, 12519.

[46] M. Skwarczynski, G. Zhao, J. C. Boer, V. Ozberk, J. G. Cruz, A. K. Giddam, Z. G. Khalil, M. Pandey, M. A. Shibu, W. M. Hussein, R. J. Nevagi, M. R. Batzloff, J. W. Wells, R. J. Capon, M. Plebanski, M. F. Good, I. Toth, Sci. Adv. 2020, 6, eaax2285.

[47] B. M. Friedrich, D. W. Beasley, J. S. Rudra, Vaccine 2016, 34, 5479.

[48] P. Leblanc, L. Moise, C. Luza, K. Chantaralawan, L. Lezeau, J. Yuan, M. Field, D. Richer, C. Boyle, W. D. Martin, J. B. Fishman, E. A. Berg, D. Baker, B. Zeigler, D. E. Mais, W. Taylor, R. Coleman, H. S. Warren, J. A. Gelfand, A. S. De Groot, T. Brauns, M. C. Poznansky, Hum. Vaccines Immunother. 2014, 10, 3022.

[49] N. Wang, J. Shang, S. Jiang, L. Du, Front. Microbiol. 2020, 11, 298. 